\title{
Transatlantica
}

Revue d'études américaines. American Studies Journal

\section{Colloque « Regards croisés sur San Francisco », Multidisciplinary Perspectives on the City by the Bay,}

Université de Provence, 20-21 mars 2009.

Pierre-François Peirano

\section{(2) OpenEdition}

Journals

Édition électronique

URL : https://journals.openedition.org/transatlantica/4341

DOI : $10.4000 /$ transatlantica.4341

ISSN : $1765-2766$

\section{Éditeur}

Association française d'Etudes Américaines (AFEA)

\section{Référence électronique}

Pierre-François Peirano, "Colloque « Regards croisés sur San Francisco », Multidisciplinary

Perspectives on the City by the Bay, », Transatlantica [En ligne], 1 | 2009, mis en ligne le 02 juillet 2009, consulté le 15 septembre 2021. URL : http://journals.openedition.org/transatlantica/4341 ; DOI : https://doi.org/10.4000/transatlantica.4341

Ce document a été généré automatiquement le 15 septembre 2021.

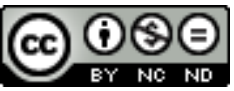

Transatlantica - Revue d'études américaines est mise à disposition selon les termes de la licence Creative Commons Attribution - Pas d'Utilisation Commerciale - Pas de Modification 4.0 International 


\section{Colloque « Regards croisés sur San Francisco », Multidisciplinary Perspectives on the City by the Bay,}

Université de Provence, 20-21 mars 2009.

\section{Pierre-François Peirano}

\section{Liste des intervenants.}

- Bolton, Marie (Université Clermont-Ferrand-II).

- Chauvin, Serge (Université Paris-X).

- Chazalon, Elodie (Université Montpellier-III).

- Cottenet, Cécile (Université de Provence).

- Delanoë-Brun, Emmanuelle (Université Paris-VII).

- Gorrha-Gobin, Cynthia (CNRS).

- Grassy, Elsa (Université Paris-IV).

- Graves, Matthew (Université de Provence).

- Ipp, Maia (City Lights Publishers).

- Jacob, François (Université de Provence).

- Lemaire, Janine (Université de Provence).

- Leriche, Frédéric (Université Toulouse-II).

- Lippert, Amy (Berkeley University).

- Massu, Claude (Université Paris-I).

- Mathé, Sylvie (Université de Provence).

- Matsumoto, Yuko (Université de Chuo, Japon).

- Nascimbene, Yan, artiste illustrateur.

- Pacini, Peggy (Institut Catholique de Paris).

- Paquet-Deyris, Anne-Marie (Université de Rouen).

- Peirano, Pierre-François (Université de Provence).

- Raffenne, Coralie (Université d'Orléans).

- Reynès-Delobel, Anne (Université de Provence).

- Rubin, Jasper (San Francisco State University). 
- Smorag, Pascale (Université de Franche-Comté).

- Tromble, Meredith (San Francisco Art Institute).

-Walker, Richard A. (University College, Berkeley).

2 Ce colloque, dont le caractère multidisciplinaire était reflété par le titre anglais, s'est tenu à l'IUFM d'Aix-en-Provence les 20 et 21 mars 2009. Placé sous l'égide de l'équipe "Aire Culturelle Nord-Américaine » du LERMA (Laboratoire d'Etude et de Recherche sur le Monde Anglophone) et organisé par Hélène Christol, Professeur Emérite à l'Université de Provence, Sylvie Mathé, Professeur à l'Université de Provence, Sophie Vallas, Maître de Conférences à l'Université de Provence, et Richard Phelan, Maître de Conférences à l'Université de Provence, il se situe dans la continuité d'un précédent colloque consacré à Chicago en 2002, ainsi que dans la droite ligne des thèmes privilégiés du LERMA, à savoir la représentation de l'espace - l'espace urbain, plus particulièrement, dans le cas de ce colloque.

3 Après une allocution de Mme Kathleen Riley, Consul des Etats-Unis à Marseille, et l'ouverture du colloque par M. le Professeur Jean Viviès, responsable du LERMA, et M. le Professeur Gérard Hugues, responsable de l'équipe "Aire Culturelle NordAméricaine", la première demi-journée a été consacrée à la géographie de San Francisco et de sa région. Ce choix permettait ainsi d'éclairer le caractère si particulier de cette ville, que l'on retrouve dans d'autres domaines mis en valeur ensuite. Après l'étude toponymique proposée par Pascale Smorag, les rapports entre l'économie créative et l'urbanisation ont été analysés par Frédéric Leriche et Jasper Rubin, avant que la communication de Richard A. Walker n'élargisse le champ de vision en traitant du mouvement environnemental dans la région de San Francisco. Lorsque l'on parle de géographie dans cette région, le risque de tremblements de terre doit également être évoqué : Marie Bolton a mêlé ce thème à l'urbanisme en établissant un parallèle entre les éphémères Earthquake Cottages, construits après le séisme de 1906, et le modèle d'habitation proposé aux habitants de la Louisiane après l'ouragan Katrina. Certains quartiers de la ville devinrent des foyers de militantisme et de contestation, deux thèmes qui ont fait l'objet des deux interventions suivantes. Celle de Janine Lemaire traitait du militantisme amérindien dans les années 1960, tandis que celle d'Elodie Chazalon s'interrogeait sur le rôle du cadre naturel de San Francisco en tant que scène ou podium pour le mouvement hippie.

La conférence plénière de Cynthia Gorrha-Gobin, directrice de recherches au CNRS, ouvrit la deuxième demi-journée et élabora la synthèse entre la qualité du paysage urbain de San Francisco - en dépit des risques naturels -, l'importance de la métropole et sa place privilégiée dans les réseaux d'influence à l'échelle fédérale. Mais, si l'on mentionne "l'influence", les liens entre San Francisco et d'autres villes, situées de l'autre côté de l'Océan Pacifique, se doivent d'être soulignés et explicités. Yuko Matsumoto a ainsi consacré son intervention à l'influence des communautés originaires d'Extrême-Orient, puis Matthew Graves a analysé les relations entre San Francisco et Sydney sous l'angle des commémorations d'événements unissant symboliquement les deux métropoles - tels que la signature d'alliances militaires ou l'afflux de populations au cours des mouvements migratoires. Après une courte pause, les Victory Gardens, forme d'art public typique de la ville, furent abordés par Meredith Tromble, du San Francisco Art Institute, puis Claude Massu s'est penché sur l'architecture contemporaine à travers l'exemple du De Young Museum, conçu en 2005, établissant un lien entre le " paysage intérieur » du musée et le cadre naturel de la ville. La conférence plénière de 
Yan Nascimbene, artiste illustrateur, a clôturé cette demi-journée. Un regard original sur la métropole cosmopolite qu'est San Francisco a été porté par l'intervenant, fort de son expérience personnelle et de ses fréquents déplacements entre l'Italie, la France et la Californie.

La deuxième journée fut tout d'abord consacrée à la place qu'occupe San Francisco dans différents domaines artistiques. Après une communication d'Elsa Grassy, consacrée au San Francisco Sound, celle de François Jacob traita de la Rat Bastard Protective Association. Puis Serge Chauvin présenta l'utilisation du cadre naturel de la ville et de ses rues en pente dans de nombreux films noirs des années 1950, avant que les intervenants suivants ne se concentrent sur des films particuliers. Ainsi, Pierre-François Peirano a traité de la représentation de San Francisco dans Dark Passage (1947), film noir réunissant Humphrey Bogart et Lauren Bacall, avant qu'Anne-Marie Paquet-Deyris ne se penche sur la topographie de la ville dans le film San Francisco (1936), célèbre pour sa scène $\mathrm{du}$ tremblement de terre. Coralie Raffenne termina ce cycle en examinant l'esthétique de la ville dans Vertigo (1958), l'une des œuvres majeures d'Alfred Hitchcock.

6 La dernière demi-journée s'ouvrit par la conférence plénière de Maia Ipp, écrivain et éditrice de la célèbre maison City Lights, qui fit un historique de la fondation de cette librairie et de son rôle grandissant dans le paysage littéraire de San Francisco. Cette conférence ouvrit ainsi la voie à la littérature. Après le passage par l'iconographie assuré par Amy Lippert et son étude du rôle de la photographie et d'autres formes d'art visuel dans le San Francisco du XIXe siècle, Cécile Cottenet traita de la revue The Overland Monthly et de la description de la ville sous la plume de son rédacteur en chef, Brian Harte. Après une communication d'Emmanuelle Delanoë-Brun sur la San Francisco fantasmée de Dashiell Hammett - laquelle établissait des correspondances avec le film noir -, Sylvie Mathé relata la quête oedipienne au centre du roman The Crying of Lot 49, de Thomas Pynchon, dans lequel émerge une bipolarité entre San Francisco et son pendant fictif, San Narciso. Réminiscence du thème de l'urbanisme, l'intervention d'Anne Reynès-Delobel, consacrée à Kay Boyle, nous plongea dans les impressions contrastées de la poétesse face à ce paysage urbain si particulier. Enfin, comment ne pas parler de San Francisco en littérature sans mentionner Jack Kerouac? Peggy Pacini combla ce manque et conclut ce colloque en analysant la peinture de la ville dans Big Sur, dans lequel elle devient l'incarnation d'une "dernière Frontière " pour le célèbre écrivain.

7 Les différentes conférences et interventions ont été regroupées autour de thèmes bien définis, à partir desquels ont émergé plusieurs constantes. Le cadre naturel de la Baie de San Francisco joue un grand rôle dans le façonnement de l'identité de la ville laquelle se retrouve dans le domaine de la musique -, mais cette idée déjà répandue fut approfondie à travers l'étude de manifestations ou d'œuvres souvent méconnues. Il est possible de se demander si San Francisco ne constitue pas elle-même une œuvre d'art à proprement parler, où le décor extérieur se reflète à l'intérieur des musées et autres habitations - c'est le cas dans de nombreux films noirs, dans lesquels les lieux sont même assimilés à des labyrinthes. L'espace de la ville devient même une source d'inspiration pour l'artiste, comme le prouvent les exemples des Victory Gardens ou des illustrations de Yan Nascimbene. Cet aspect contribua à enrichir les communications consacrées aux mouvements artistiques et contestataires qui s'y développèrent. Quant à la perspective environnementaliste, elle a fourni un angle d'attaque novateur, 
rappelant que la San Francisco Bay Area n'était pas seulement caractérisée par une urbanité galopante. Si l'accent fut mis sur les différents éléments qui contribuèrent au formidable creuset d'influences qu'est San Francisco, les différentes interventions ont également mené à s'interroger sur le rayonnement de la ville hors des frontières de la Californie et des Etats-Unis. Le développement de la librairie City Lights en constitue la métaphore et l'étude de diverses œuvres littéraires ou magazines a permis de porter un regard nouveau sur la ville, dont la géographie devient le vecteur de l'imaginaire.

Ainsi, ce colloque donna lieu à des échanges fort constructifs et à l'établissement de nombreux liens entre les différents thèmes, alimentant son caractère multidisciplinaire. La présence de personnes appartenant à des milieux divers - tels que l'art ou l'édition - a conféré une indéniable richesse à ces "regards croisés ». Ils firent émerger l'image unique de San Francisco dans la culture et la pensée américaines, sans oublier l'imaginaire que fait naître cette métropole. Une phrase de l'écrivain William Saroyan définit San Francisco ainsi : "No city invites the heart to come to life as San Francisco does". Ce colloque en fut la parfaite illustration.

INDEX

Thèmes: Actualité de la recherche

\section{AUTEUR}

PIERRE-FRANÇOIS PEIRANO

Doctorant, ATERUniversité de Provence, Université du Sud Toulon-Var 